\title{
Riemannian Twistor Spaces and Holonomy Groups
}

\author{
Francis Burstall \\ From Twistors in Mathematics and Physics, C.U.P. (1990), pp. 53--70
}

\section{Introduction}

The essence of the twistor programme is to encode the differential geometry of a manifold by holomorphic data on some auxiliary complex space (a twistor space). Thus problems in (pseudo) Riemannian Geometry are converted into (hopefully soluble) problems in Complex Analysis or Algebraic Geometry. As examples of such constructions, let us mention:

- The Penrose fibration $\mathbf{C} P^{3} \rightarrow \mathbf{H} P^{1}=S^{4}$ encodes a large part of the geometry of the 4 -sphere. For instance, instanton solutions to the Yang-Mills equations on $S^{4}$ pull back to holomorphic bundles on $\mathbf{C} P^{3}$ [30]. This forms the basis of the Atiyah-Drinfeld-HitchinManin classification of the instantons [1]. Again, horizontal curves in $\mathbf{C} P^{3}$ project onto branched minimal surfaces in $S^{4}$. Using this Bryant [7] was able to establish the existence of embedded minimal surfaces of arbitrary genus in $S^{4}$.

- In a similar vein, consider the homogeneous fibrations

$$
\frac{\mathrm{U}(n+1)}{\mathrm{U}(r) \times \mathrm{U}(1) \times \mathrm{U}(n-r)} \longrightarrow \frac{\mathrm{U}(n+1)}{\mathrm{U}(1) \times \mathrm{U}(n)}
$$

of flag manifolds over projective spaces. Again horizontal holomorphic curves in the flag manifolds project onto branched minimal surfaces (harmonic maps) in $\mathbf{C} P^{n}$. Further, all minimal 2-spheres in $\mathbf{C} P^{n}$ arise in this way. This is the starting point of the classification theorem for harmonic 2 -spheres in complex projective spaces [9, 14, 16, 18].

- Finally we briefly consider an example with a different flavour. The space of geodesics in $\mathbf{R}^{3}$ may be identified with the holomorphic line bundle $T^{1,0} \mathbf{C} P^{1}$. Then magnetic monopoles on $\mathbf{R}^{3}$ can be shown to correspond to certain algebraic curves (spectral curves) in $T^{1,0} \mathbf{C} P^{1}$ [20]. Somewhat more transparently, minimal surfaces in $\mathbf{R}^{3}$ correspond to algebraic curves (essentially without restriction) in $T^{1,0} \mathbf{C} P^{1}$ and this provides a geometrical interpretation of the Weierstrass representation formulae [20,27].

In this article we shall concentrate on the construction of twistor spaces rather than their applications. We shall describe fibrations of complex manifolds over Riemannian manifolds that generalise those in the first two of the preceding examples. For applications to the theory of harmonic maps, the Reader is referred to the survey articles [10, 11, 29].

\section{The bundle of almost complex structures}

The first two examples listed above have much in common: in both cases the twistor space is a complex manifold fibred over the Riemannian manifold of interest. The fibration is not 
holomorphic (even when this makes sense) but the fibres are complex submanifolds of the twistor space. Let us now see how we might build such fibrations over more general Riemannian manifolds.

So let $N$ be a $2 n$-dimensional Riemannian manifold. We may at least construct such a fibration of an almost complex manifold over $N$ as follows: let $\pi: J(N) \rightarrow N$ be the bundle of almost Hermitian structures of $N$. Thus the fibre at $x \in N$ is

$$
J_{x}(N)=\left\{j \in \operatorname{End}\left(T_{x} N\right): j^{2}=-1, j \text { skew-symmetric }\right\} .
$$

This bundle is associated to the orthonormal frame bundle of $N$ with typical fibre $J\left(\mathbf{R}^{2 n}\right)=$ $\mathrm{O}(2 n) / \mathrm{U}(n)$ which is a Hermitian symmetric space (in fact it is two disjoint copies of the compact irreducible Hermitian symmetric space $\mathrm{SO}(2 n) / \mathrm{U}(n))$. In particular, the typical fibre has an $\mathrm{O}(2 n)$-invariant complex structure and thus the vertical distribution $\mathcal{V}=$ ker $d \pi$ inherits an almost complex structure $J^{\mathcal{V}}$. The Levi-Civita connection on the orthonormal frame bundle induces a horizontal distribution $\mathcal{H}$ on $J(N)$ so that we have a splitting

$$
T J(N)=\mathcal{V} \oplus \mathcal{H}
$$

with $d \pi$ giving an isomorphism between $\mathcal{H}$ and $\pi^{-1} T N$. This enables us to define a tautological almost complex structure $J^{\mathcal{H}}$ on $\mathcal{H}$ by

$$
J_{j}^{\mathcal{H}}=j
$$

and adding this to $J^{\mathcal{V}}$ gives us an almost complex structure $\mathcal{J}=J^{\mathcal{V}} \oplus J^{\mathcal{H}}$ on $J(N)$. By construction, the fibres of $\pi$ are almost complex submanifolds with respect to $\mathcal{J}$.

Before going any further, let us remark that if we make a conformal change of metric on $N$, the bundle $J(N)$ remains unchanged although the horizontal distribution $\mathcal{H}$ will vary. However, despite this, it can be shown that the almost complex structure $\mathcal{J}$ is independent of the choice of metric within a conformal class. Thus our construction may be viewed as one in Conformal Geometry but we shall not pursue this here.

Having got our almost complex structure, it is natural to ask whether or not it is integrable so that $J(N)$ is an honest complex manifold. For this, of course, it is necessary and sufficient that the Nijenhuis tensor $N^{\mathcal{J}}$ of $\mathcal{J}$ vanish. The obstruction to this vanishing lies in the curvature tensor of $N[22]$ :

Theorem 2.1 Let $j \in J(N)$ with $\sqrt{-1}$-eigenspace $T^{+} \subset T_{\pi(j)} N^{\mathrm{C}}$. Let $R$ denote the Riemann curvature tensor of $N$. Then $N^{\mathcal{J}}$ vanishes at $j$ if and only if

$$
R\left(T^{+}, T^{+}\right) T^{+} \subset T^{+} .
$$

Thus $\mathcal{J}$ is integrable if (1) holds for all maximally isotropic subspaces $T^{+}$of $T N^{\mathbf{C}}$. This is a condition on the curvature tensor that can be analysed in terms of the representation theory of $\mathrm{O}(2 n)$ on the space of curvature tensors and one concludes:

Corollary 2.2 $\mathcal{J}$ is integrable if and only if the Weyl tensor of $R$ vanishes identically (i.e. $N$ is locally conformally flat).

Thus $J(N)$ is a complex manifold only in extremely restricted circumstances. The moral to be drawn from this is that $J(N)$ is "too big" in general for $\mathcal{J}$ to be integrable. It is therefore appropriate to seek subbundles of $J(N)$ picked out by the geometry of $N$ in the hope that some of these are complex manifolds. One way to do this is is to restrict attention to those elements of $J(N)$ that are compatible with the holonomy of $N$. It is to this that we now turn. 


\section{Reduction to the holonomy group}

So let our $2 n$-dimensional manifold $N$ have holonomy group $K$ and let $P \rightarrow N$ denote the holonomy bundle i.e the reduction of the orthonormal frame bundle of $N$ to $K$. The typical fibre $J\left(\mathbf{R}^{2 n}\right)$ of $J(N)$ decomposes into a disjoint union of $K$-orbits and, correspondingly, $J(N)$ decomposes into a disjoint union of subbundles, each one associated to $P$ with such an orbit as typical fibre. We now investigate whether any of these subbundles are complex manifolds with respect to $\mathcal{J}$.

There are two aspects to this question: firstly, we are doomed to failure unless our subbundle is $\mathcal{J}$-invariant i.e is an almost complex submanifold of $(J(N), \mathcal{J})$. Since our subbundles are associated to the holonomy bundle $P$, their inclusion into $J(N)$ preserves horizontal distributions which are therefore $\mathcal{J}$-invariant. Thus, for our subbundle to be $\mathcal{J}$-invariant, it is necessary and sufficient that the fibres be $\mathcal{J}$-invariant or, equivalently, that the corresponding $K$-orbit in $J\left(\mathbf{R}^{2 n}\right)$ be a holomorphic submanifold. Secondly, once we have a $\mathcal{J}$-invariant subbundle, we must ascertain whether the induced almost complex structure thereon (also called $\mathcal{J}$ ) is integrable. According to O'Brian-Rawnsley [22], this happens precisely when the Nijenhuis tensor of $\mathcal{J}$ vanishes on the subbundle. In view of (2.1), this is a condition that can be analysed in terms of the representation theory of $K$ on the space of curvature tensors of metrics with holonomy $K$. This last topic is dealt with in some detail in the book [25] which is our source for most of the information about holonomy groups that we need below.

We begin by assuming that $N$ is orientable so that $K \subseteq \mathrm{SO}(2 n)$. Of course, $\mathrm{SO}(2 n)$ acts transitively on connected components of $J\left(\mathbf{R}^{2 n}\right)$ and so we have two orbits both of which are complex submanifolds. The corresponding subbundles are denoted $J_{+}(N)$ and $J_{-}(N)$ with $J_{+}(N)$ consisting of those almost Hermitian structures $j$ compatible with the orientation in the sense that $x_{1} \wedge j x_{1} \wedge \ldots \wedge x_{n} \wedge j x_{n}$ is a non-negative multiple of the volume form for any vectors $x_{1}, \ldots, x_{n} \in T_{\pi(j)} N$. We may now examine the integrability of $\mathcal{J}$ on $J_{+}(N)$ and $J_{-}(N)$ separately. If $2 n \geq 6$, we get nothing new and $\mathcal{J}$ is integrable precisely when the Weyl tensor vanishes [15, 22]. However, when $N$ is 4-dimensional, it is a celebrated result of Singer-Thorpe [26] that the Weyl tensor splits into two parts under the action of $\mathrm{SO}(4)$ and then each part constitutes the obstruction to the integrability of $\mathcal{J}$ on one of $J_{+}(N)$ or $J_{-}(N)$ [2]. In summary, we have

Theorem 3.1 If $2 n \geq 6, \mathcal{J}$ is integrable on $J_{+}(N)$ or $J_{-}(N)$ if and only if $N$ is locally conformally flat. If $2 n=4, \mathcal{J}$ is integrable on $J_{+}(N)$ if and only if $N$ is anti-self-dual and on $J_{-}(N)$ if and only if $N$ is self-dual.

To go further, we must consider $N$ with holonomy strictly contained in $\mathrm{SO}(2 n)$. To simplify matters, we shall suppose that $N$ is simply-connected, oriented and irreducible. If $N$ is not a symmetric space, the classification of Berger [4] tells us that the only possiblities for $K$ are $\mathrm{SO}(2 n), \mathrm{U}(n), \mathrm{SU}(n), \operatorname{Sp}(1) \operatorname{Sp}\left(\frac{n}{2}\right), \operatorname{Sp}\left(\frac{n}{2}\right)$ or $\operatorname{Spin}(7)$ (this last acting on $\mathbf{R}^{8}$ via the spin representation). Before discussing each of these in turn, let us establish some generalities about $K$-orbits in $J\left(\mathbf{R}^{2 n}\right)$.

Firstly, an analysis of the complex structure of $J\left(\mathbf{R}^{2 n}\right)$ (c.f. [22]) provides us with the following criterion for the holomorphicity of a $K$-orbit.

Lemma 3.2 The K-orbit of $j \in J\left(\mathbf{R}^{2 n}\right)$ is a complex submanifold of $J\left(\mathbf{R}^{2 n}\right)$ if and only if the 
Lie algebra $\mathbf{k} \subset \mathrm{so}(2 n)$ of $K$ satisfies

$$
[[\mathbf{k}, j], j]=[\mathbf{k}, j]
$$

The second fact we use about a holomorphic $K$-orbit is

Proposition 3.3 If the $K$-orbit of $j$ is a holomorphic submanifold then the stabiliser of $j$ is the centraliser of a torus in $K$ and, in particular, contains a maximal torus in $K$.

To prove this, observe that a connected component of the Kähler manifold $J\left(\mathbf{R}^{2 n}\right)$ is an adjoint orbit of $\mathrm{SO}(2 n)$ and the inclusion may be regarded (after identifying $\mathrm{so}(2 n)$ with its dual) as the moment map for the (isometric, holomorphic and hence symplectic) action of $\mathrm{SO}(2 n)$. Now a holomorphic $K$-orbit acquires a $K$-invariant Kähler structure from $J\left(\mathbf{R}^{2 n}\right)$ and hence, by the functoriality of the moment map construction, is an adjoint orbit of $K$. This suffices to establish (3.3).

For our first application of these results, let us suppose that $N$ is a Kähler manifold with $K=\mathrm{U}(n)$. We start by considering the situation in a typical fibre: let $j_{0} \in J\left(\mathbf{R}^{2 n}\right)$ be the complex structure corresponding to the Kähler structure. Then $j_{0}$ lies in the centre of $\mathrm{u}(n)$ and so lies in any maximal toral subalgebra of $\mathrm{u}(n)$. It is now easy to conclude from (3.2) and (3.3) that the $\mathrm{U}(n)$ orbit of $j$ is a holomorphic submanifold of $J\left(\mathbf{R}^{2 n}\right)$ if and only if $j$ commutes with $j_{0}$. This provides us with a simple geometric interpretation of the holomorphic orbits: let $T$ be the $\sqrt{-1}$-eigenspace of $j_{0}$; if $j$ commutes with $j_{0}$ then we have an orthogonal decomposition of $T$ into eigenspaces of $j$ so that

$$
T=T^{\prime} \oplus T^{\prime \prime}
$$

with $j=j_{0}$ on $T^{\prime}$ and $j=-j_{0}$ on $T^{\prime \prime}$. Conversely, an such splitting of $T$ determines a $j$ commuting with $j_{0}$ by the above prescription. Thus the holomorphic $\mathrm{U}(n)$-orbits are just the Grassmannians $G_{r}(T)$ of $r$-dimensional complex subspaces of $T$ for $r=0,1, \ldots, n$.

The corresponding subbundles are just the Grassmannian bundles $G_{r}\left(T^{1,0} N\right)$ embedded in $J(N)$ by the map

$$
W \longmapsto j_{W}= \begin{cases}\sqrt{-1} & \text { on } W \oplus\left(\bar{W}^{\perp} \cap T^{0,1} N\right) \\ -\sqrt{-1} & \text { on } \bar{W} \oplus\left(W^{\perp} \cap T^{1,0} N\right) .\end{cases}
$$

These Grassmannian bundles with the almost complex structure $\mathcal{J}$ inherited from $J(N)$ where considered by O'Brian-Rawnsley (op. cit.) who showed that for $1 \leq r \leq n-1, \mathcal{J}$ is integrable on $G_{r}\left(T^{1,0} N\right)$ if and only if the Bochner tensor of $N$ vanishes. Of course, $G_{0}\left(T^{1,0} N\right)$ and $G_{n}\left(T^{1,0}\right)$ are just the images of the sections of $J(N)$ defined by the Kähler structure and its negative and so always have integrable $\mathcal{J}$.

As an example of this development, let us take $N$ to be a complex projective space $\mathbf{C} P^{n}$. In this case the Bochner tensor vanishes so that $\mathcal{J}$ is integrable on each $G_{r}\left(T^{1,0} \mathbf{C} P^{n}\right)$. In fact, $G_{r}\left(T^{1,0} \mathbf{C} P^{n}\right)$ is naturally isomorphic to the flag manifold $\mathrm{U}(n+1) / \mathrm{U}(r) \times \mathrm{U}(1) \times \mathrm{U}(n-r)$ and the bundle projection is just the homogeneous fibration discussed in the introduction .

Finally we remark that the Grassmannian bundles always acquire an integrable complex structure from the holomorphic frame bundle of $N$. For this complex structure the bundle projection is holomorphic and so does not coincide with our $\mathcal{J}$ unless the fibres are zero-dimensional (i.e. $r=0$ or $n$ ). 
If $N$ is Ricci-flat Kähler so that $K=\mathrm{SU}(n)$ we get nothing new: the only holomorphic orbits are the Grassmannian orbits we have already discovered. However, in this case, the Bochner tensor constitutes the whole of the curvature tensor and so unless $N$ is flat, $\mathcal{J}$ is not integrable for $1 \leq r \leq n-1$.

Examples Let us illustrate the situation by considering the two 4-dimensional symmetric spaces. Firstly, let $N=S^{4}$ with holonomy $\mathrm{SO}(4)$. Of course $S^{4}$ is conformally flat so that both $J_{+}\left(S^{4}\right)$ and $J_{-}\left(S^{4}\right)$ are both complex manifolds with respect to $\mathcal{J}$. Indeed, both these spaces are isometrically biholomorphic to $\mathrm{SO}(5) / \mathrm{U}(2) \cong \mathbf{C} P^{3}$ and the projections are just the Penrose fibration and its post-composition with the antipodal map.

Now let $N=\mathbf{C} P^{2}$ which has holonomy $\mathrm{U}(2)$ and is self-dual but not conformally flat. From (3.1) we conclude that $\mathcal{J}$ is integrable on $J_{-}\left(\mathbf{C} P^{2}\right)$ but not on $J_{+}\left(\mathbf{C} P^{2}\right)$. In fact, we may deduce this result from the above discussion since it is easy to show that $j \in J_{-}\left(\mathbf{R}^{4}\right)$ if and only if $\left[j_{0}, j\right]$ vanishes and $j \neq \pm j_{0}$. This provides an isomorphism of $J_{-}\left(\mathbf{R}^{4}\right)$ with $G_{1}(T) \cong \mathbf{C} P^{1}$ and so we may identify $J_{-}\left(\mathbf{C} P^{2}\right)$ with the flag manifold $P\left(T^{1,0} \mathbf{C} P^{2}\right)$. The integrability of $J_{-}\left(\mathbf{C} P^{2}\right)$ now follows from the fact that the Bochner tensor of $\mathbf{C} P^{2}$ vanishes (for a Kähler surface, this is the same as being self-dual). In $J_{+}\left(\mathbf{C} P^{2}\right)$ on the other hand, the only subbundles on which $\mathcal{J}$ is integrable are the two copies of $\mathbf{C} P^{2}$ corresponding to the Kähler structure and its negative.

Consider now the case of quaternionic Kähler manifolds, that is manifolds with holonomy contained in $\operatorname{Sp}(1) \operatorname{Sp}(k)$ [24] where $2 n=4 k$. Geometrically, this means that there is a parallel subbundle $\mathcal{C}$ of $\operatorname{End}(T N)$ locally spanned by sections $I, J, K$ which satisfy the familiar identities

$$
I^{2}=J^{2}=K^{2}=-1, I J=-J I=K \text { etc. }
$$

Following [24], we identify the representation of $\operatorname{Sp}(1) \operatorname{Sp}(k)$ on a complexified tangent space $T$ as follows

$$
T \cong H \otimes_{\mathbf{C}} E
$$

where $H$ and $E$ are the 2 and $2 k$ complex dimensional representations of $\operatorname{Sp}(1)$ and $\operatorname{Sp}(k)$ respectively. In this setting the real structure on $T$ is the tensor product of the quaternionic structures $j_{H}$ and $j_{E}$ on $H$ and $E$.

Let us now suppose that $K=\operatorname{Sp}(1) \operatorname{Sp}(k)$ and consider $K$-orbits in $J\left(\mathbf{R}^{4 k}\right)$. We begin by recalling that $H$ and $E$ carry invariant complex symplectic forms $\omega_{H}$ and $\omega_{E}$ whose tensor product gives the metric on $T$. Using (3.2) and (3.3), one may now deduce that there are three kinds of holomorphic orbit: the first and most important of which arise by choosing a complex line $H^{+} \subset H$ and considering the almost complex structure with $\sqrt{-1}$-eigenspace

$$
T^{1,0}=H^{+} \otimes E .
$$

This is Hermitian since $\omega_{H}$ necessarily vanishes on $H^{+}$. These almost complex structures comprise a single orbit $P(H) \cong \mathrm{Sp}(1) / \mathrm{U}(1)$ which can also be described as the elements in a fibre of $\mathcal{C}$ with square -1 . This is just the sphere of radius 2 (with respect to the trace norm) in $\mathcal{C}$.

Dual to this orbit is one composed of almost complex structures with $\sqrt{-1}$-eigenspace

$$
T^{1,0}=H \otimes E^{+}
$$

where $E^{+} \subset E$ is a Lagrangian subspace. $\operatorname{Sp}(k)$ acts transitively on such $E^{+}$and we get a single orbit isomorphic to $\operatorname{Sp}(k) / \mathrm{U}(k)$. Finally, there is a class of holomorphic orbits obtained 
by mixing these constructions. Pick $H^{+} \subset H$ and $E^{+} \subset E$ with $E^{+}$isotropic for $\omega_{E}$ but not maximally isotropic (Lagrangian). Then we have a $j_{E}$ invariant decomposition

$$
E=E^{+} \oplus E^{0} \oplus E^{-}
$$

with $E^{0}$ stable under $j_{E}$ and $j_{E} E^{+}=E^{-}$. Then the orbit of the $j$ with $\sqrt{-1}$-eigenspace

$$
\left(H^{+} \otimes E^{0}\right) \oplus\left(H \otimes E^{+}\right)
$$

is holomorphic and is isomorphic to $\operatorname{Sp}(1) \times \operatorname{Sp}(k) / \mathrm{U}(1) \times \mathrm{U}(r) \times \operatorname{Sp}(k-r)$ where $\operatorname{dim}_{\mathbf{C}} E^{+}=r$. These three kinds of orbit exhaust the holomorphic orbits of $\operatorname{Sp}(1) \operatorname{Sp}(k)$ in $J\left(\mathbf{R}^{4 k}\right)$.

Turning to the integrability of $\mathcal{J}$ on the corresponding subbundles, the first orbit considered corresponds to the radius 2 sphere bundle of $\mathcal{C}$ and it is a theorem of Salamon [24] that $\mathcal{J}$ is always integrable on this bundle. As for the remaining subbundles, the analysis in [25] of the curvature of quaternionic Kähler metrics can be used to show that $\mathcal{J}$ is integrable precisely when $N$ is locally symmetric and so locally isometric to quaternionic projective space.

In case that $K=\operatorname{Sp}(k)$, the bundle $\mathcal{C}$ is flat and so spanned by global parallel sections $I, J, K$ satisfying (2). Such manifolds are called hyper-Kähler manifolds and have been the object of much recent study (c.f. [25]). From our point of view though, we get nothing new. $\operatorname{Sp}(k)$ acts trivially on $H$ and so the sphere bundle of $\mathcal{C}$ decomposes into the images of the global parallel sections $a I+b J+c K$ where $a^{2}+b^{2}+c^{2}=1$ and, of course, $\mathcal{J}$ is integrable on all of these. As for the remaining subbundles, $\mathcal{J}$ is not integrable on any of these unless $N$ is flat.

Finally, we must consider the case $K=\operatorname{Spin}(7)$ acting on $\mathbf{R}^{8}$ by the spin representation. It turns out that there are two holomorphic orbits in $J\left(\mathbf{R}^{8}\right)$ isomorphic to $\mathrm{SO}(7) / \mathrm{U}(3)$ and the 5 -quadric $\mathrm{SO}(7) / \mathrm{SO}(2) \times \mathrm{SO}(5)$. However, the form of the curvature tensor prevents $\mathcal{J}$ from being integrable on either of the corresponding subbundles.

This concludes the analysis of $J(N)$ when $N$ is not locally symmetric. We have seen that with the notable exception of the quaternionic Kähler manifolds, honest complex submanifolds of $J(N)$ are pretty hard to come by. However, the situation is quite different for symmetric spaces. Indeed, using (2.1), (3.2) and (3.3), one can prove

Lemma 3.4 If $N$ is locally symmetric and irreducible, then the $K$-orbit of $j \in J\left(\mathbf{R}^{2 n}\right)$ is a complex submanifold if and only if the Nijenhuis tensor of $\mathcal{J}$ vanishes on the corresponding subbundle of $J(N)$.

Thus in this situation our subbundle has integrable $\mathcal{J}$ as soon as it is an almost complex manifold and one would expect to find a good supply of complex submanifolds of $J(N)$. This is indeed the case as we shall now see.

\section{$4 \quad$ Flag manifolds and symmetric spaces}

We now suppose that $N$ is an irreducible $2 n$-dimensional Riemannian symmetric space. Thus we may realise $N$ as a coset space $N=G / K$ with $G^{\tau} \subset K \subset\left(G^{\tau}\right)_{0}$ for some involution $\tau$ of $G$. Now $K$ is (a covering of) the holonomy group of $N$ and similarly the coset fibration $G \rightarrow G / K$ covers the holonomy bundle $P \rightarrow N$. In this setting, $J(N)$ is associated to $G$ :

$$
J(N) \cong G \times_{K} J\left(\mathbf{R}^{2 n}\right)
$$


and if $K / H$ is a $K$-orbit in $J\left(\mathbf{R}^{2 n}\right)$ then the corresponding subbundle is $G \times_{K} K / H=G / H$ and the projection is just the coset fibration. Thus we see that the subbundles of $J(N)$ that we are considering are just the orbits of $G$ in $J(N)$. We may now rephrase (3.4) as follows:

Proposition 4.1 Let $j \in J(N)$. Then $G \cdot j$ is an almost complex submanifold of $J(N)$ on which $\mathcal{J}$ is integrable if and only if $j$ lies in the zero-set of the Nijenhuis tensor $N^{\mathcal{J}}$.

This focusses our attention on the zero-set of $N^{\mathcal{J}}$ which we denote by $Z$. In favourable circumstances, the structure of this set can be completely described. We begin by assuming that $N$ is of compact type so that $G$ is compact and semi-simple. We also assume that $N$ is inner i.e. that $\tau$ is an inner involution of $G$ or, equivalently, that $\operatorname{rank} G=\operatorname{rank} K$. The class of inner symmetric spaces include the even-dimensional spheres, the Hermitian symmetric spaces, the quaternionic Kähler symmetric spaces and indeed all symmetric $G$-spaces for $G=\mathrm{SO}(2 n+1), \mathrm{Sp}(n), \mathrm{E}_{7}, \mathrm{E}_{8}, \mathrm{~F}_{4}$ and $\mathrm{G}_{2}$. Moreover, all inner symmetric spaces are necessarily even-dimensional and so fit into our framework.

Our assumption that $N$ be inner can also be motivated by (3.3): the stabiliser in $G$ of $j \in Z$ is the centraliser of a torus in $K$ and so contains a maximal torus of $K$; if $N$ is inner, this torus is also maximal in $G$ from which it follows that the stabiliser is the centraliser of a torus in $G$. Thus, for $N$ inner, our orbit is of the form $G / C(T)$ for some torus $T \subset G$ and is therefore a flag manifold (c.f. [31]). According to Borel [5], these exhaust the compact Kählerian $G$-spaces for $G$ semi-simple. We now have the following remarkable theorem:

Theorem 4.2 ([12]) Let $N=G / K$ be a simply-connected inner Riemannian symmetric space of compact type. Then $Z$ consists of a finite number of connected components on each of which $G$ acts transitively. Moreover, any $G$-flag manifold is realised as such an orbit for some $N$.

Remark A similar result for a certain subset of $Z$ has been proved by Bryant [8].

The proof of (4.2) requires a detour into the geometry of flag manifolds and reveals an interesting interaction between the complex geometry of flag manifolds and the real geometry of inner symmetric spaces. For this, we begin by noting that a coset space of the form $G / C(T)$ admits several invariant Kählerian complex structures in general [6]. To fix attention on just one of these, we use a complex realisation of $G / C(T)$ as follows: having fixed a complex structure, the complexified group $G^{\mathbf{C}}$ acts transitively on $G / C(T)$ by biholomorphisms with parabolic subgroups as stabilisers. Conversely, if $P \subset G^{\mathbf{C}}$ is a parabolic subgroup then the action of $G$ on $G^{\mathbf{C}} / P$ is transitive and $G \cap P$ is the centraliser of a torus in $G$. Let us examine the infinitesimal situation: let $F=G / C(T)$ be a flag manifold and let $o \in F$. We have a splitting of the Lie algebra of $G$

$$
\mathbf{g}^{\mathbf{C}}=\mathbf{h} \oplus \mathbf{m}
$$

with $\mathbf{m} \cong T_{o} F$ and $\mathbf{h}$ the Lie algebra of the stabiliser of $o$ in $G$. An invariant complex structure on $F$ induces an ad $\mathbf{h}$-invariant splitting of $\mathbf{m}^{\mathbf{C}}$ into $(1,0)$ and $(0,1)$ spaces

$$
\mathbf{m}^{\mathbf{C}}=\mathbf{m}^{+} \oplus \mathbf{m}^{-}
$$

with $\left[\mathbf{m}^{+}, \mathbf{m}^{+}\right] \subset \mathbf{m}^{+}$by integrability. One can show that $\mathbf{m}^{+}$and $\mathbf{m}^{-}$are nilpotent subalgebras of $\mathbf{g}^{\mathbf{C}}$ and in fact $\mathbf{h}^{\mathbf{C}} \oplus \mathbf{m}^{-}$is a parabolic subalgebra of $\mathbf{g}^{\mathbf{C}}$ with nilradical $\mathbf{m}^{-}$. If $P$ is the corresponding parabolic subgroup of $G^{\mathbf{C}}$ then $P$ is the stabiliser of $o$ and we obtain a biholomorphism between the complex coset space $G^{\mathbf{C}} / P$ and the flag manifold $F$. 
Conversely, let $P \subset G^{\mathbf{C}}$ be a parabolic subgroup with Lie algebra $\mathbf{p}$ and let $\mathbf{n}$ be the conjugate of the nilradical of $\mathbf{p}$ (with respect to the real form $\mathbf{g}$ ). Then $H=G \cap P$ is the centraliser of a torus and we have orthogonal decompositions (with respect to the Killing inner product)

$$
\mathbf{p}=\mathbf{h}^{\mathbf{C}} \oplus \overline{\mathbf{n}}, \mathbf{g}^{\mathbf{C}}=\mathbf{h}^{\mathbf{C}} \oplus \mathbf{n} \oplus \overline{\mathbf{n}}
$$

which define an invariant complex structure on $G / H$ realising the biholomorphism with $G^{\mathbf{C}} / P$.

The relationship between a flag manifold $F=G^{\mathbf{C}} / P$ and an inner symmetric space comes from an examination of the central descending series of $\mathbf{n}$. Recall that this is a filtration $0=\mathbf{n}_{k+1} \subset \mathbf{n}_{k} \subset \ldots \subset \mathbf{n}_{1}=\mathbf{n}$ of $\mathbf{n}$ defined by

$$
\mathbf{n}_{i}=\left[\mathbf{n}, \mathbf{n}_{i-1}\right] .
$$

We orthogonalise this filtration using the Killing inner product by setting

$$
\mathbf{g}_{i}=\mathbf{n}_{i+1}^{\perp} \cap \mathbf{n}_{i}
$$

for $i \geq 1$ and extend this to a decomposition of $\mathbf{g}^{\mathbf{C}}$ by setting $\mathbf{g}_{0}=\mathbf{h}^{\mathbf{C}}=(\mathbf{g} \cap \mathbf{p})^{\mathbf{C}}$ and $\mathbf{g}_{-i}=\overline{\mathbf{g}_{i}}$ for $i \geq 1$. Then

$$
\mathbf{g}^{\mathbf{C}}=\sum \mathbf{g}_{i}
$$

is an orthogonal decomposition with

$$
\mathbf{p}=\sum_{i \leq 0} \mathbf{g}_{i}, \mathbf{n}=\sum_{i>0} \mathbf{g}_{i} .
$$

The crucial property of this decomposition is that

$$
\left[\mathbf{g}_{i}, \mathbf{g}_{j}\right] \subset \mathbf{g}_{i+j}
$$

which can be proved by demonstrating the existence of an element $\xi \in \mathbf{h}$ with the property that, for each $i$, ad $\xi$ has eigenvalue $\sqrt{-1} i$ on $\mathbf{g}_{i}$. This element $\xi$ (necessarily unique since $\mathrm{g}$ is semi-simple) was shown to exist by Burstall-Rawnsley [12] who called it the canonical element of $\mathbf{p}$. Since ad $\xi$ has eigenvalues in $\sqrt{-1} \mathbf{Z}, \operatorname{Ad} \exp \pi \xi$ is an involution of $\mathbf{g}$ which we exponentiate to obtain an inner involution $\tau_{\xi}$ of $G$ and thus an inner symmetric space $G / K$ where $K=\left(G^{\tau_{\xi}}\right)_{0}$. Clearly, $K$ has Lie algebra given by

$$
\mathbf{k}=\mathbf{g} \cap \sum_{i} \mathbf{g}_{2 i}
$$

and so contains $H$ whence we obtain a homogeneous fibration $G / H \rightarrow G / K$ of our flag manifold over our inner symmetric space. Moreover, this fibration is essentially unique: the only ambiguity in the prescription is that several points in the symmetric space might have the same stabiliser $K$ (e.g. antipodal points on a sphere). However, the number of such points is finite and so we only get a finite number of such fibrations. We call these fibrations the canonical fibrations of $F$. To summarise:

Theorem 4.3 Let $F=G^{\mathbf{C}} / P$ be a flag manifold. Then there is a unique inner symmetric space $G$-space $N$ associated to $F$ together with a finite number of homogeneous fibrations $F \rightarrow$ $N$.

Let us emphasise that this construction depends on nothing but the conjugacy class of $\mathbf{p} \subset \mathbf{g}^{\mathbf{C}}$ and the choice of compact real form $\mathbf{g}$. Equivalently, it depends solely on the choice of invariant complex structure on $F$. 
We have now seen that every flag manifold fibres over an inner symmetric space. Conversely, it is straightforward to show [12] that every inner symmetric space is the target of the canonical fibrations of at least one flag manifold. Let us now see how this story relates to the geometry of $J(N)$.

So let $p: F \rightarrow N$ be a canonical fibration. By construction, the fibres of $p$ are complex submanifolds of $F$ and this allows us to define a fibre map $i_{p}: F \rightarrow J(N)$ as follows: at $f \in F$ we have an orthogonal splitting of $T_{f} F$ into horizontal and vertical subspaces both of which are invariant under the complex structure of $F$. Then $d p$ restricts to give an isomorphism of the horizontal part with $T_{p(f)} N$ and therefore induces an almost Hermitian structure on $T_{p(f)} N$ : this is $i_{p}(f) \in J_{p(f)} N$. Such a construction is possible whenever we have a Riemannian submersion of a Hermitian manifold with complex submanifolds as fibres. In the case at hand we have:

Proposition $4.4 i_{p}: F \rightarrow J(N)$ is a G-equivariant holomorphic embedding.

This implies that $i_{p}(F)$ is an almost complex submanifold of $J(N)$ on which $\mathcal{J}$ is integrable. Thus as a corollary of (4.1) and (4.4) we have

Corollary $4.5 i_{p}(F)$ is a $G$-orbit in $Z \subset J(N)$.

In particular, this guarantees that $Z \neq \emptyset$. Moreover, it turns out that the converse to (4.5) is true.

Theorem 4.6 ([12]) If $j \in Z \subset J(N)$ then $G \cdot j$ is a flag manifold canonically fibred over $N$. In fact, $G \cdot j=i_{p}(F)$ for some canonical fibration $p: F \rightarrow N$ of a flag manifold $F$.

For this, the main observation is the following: at $\pi(j)$, we have the symmetric decomposition

$$
\mathbf{g}=\mathbf{k} \oplus \mathbf{q}
$$

with $\mathbf{q} \cong T_{\pi(j)} N$. If $\mathbf{q}^{-}$is the $(0,1)$-space for $j$ then

$$
\left[\mathbf{q}^{-}, \mathbf{q}^{-}\right] \oplus \mathbf{q}^{-}
$$

is the nilradical of a parabolic subalgebra p. One can then show that $G \cdot j$ is equivariantly biholomorphic to the corresponding flag manifold $G^{\mathbf{C}} / P$ as described in (4.6).

We are now in a position to complete the proof of (4.2). We have seen each canonical fibration of a flag manifold gives arise to a $G$-orbit in $Z$ for some inner symmetric $G$-space $N$ and that all such orbits arise in this way. But, for fixed $G$, there are only a finite number of biholomorphism types of flag manifold (they are in bijective correspondence with the conjugacy classes of parabolic subalgebras of $\mathbf{g}^{\mathbf{C}}$ ) and each flag manifold admits but a finite number of canonical fibrations. Thus $Z$ is composed of a finite number of $G$-orbits all of which are closed and (4.2) follows. It is interesting to note that in this way we obtain a geometric interpretation of the purely algebraic construction of the canonical fibrations: they are just the restrictions of the projection $\pi: J(N) \rightarrow N$ to the various realisations of $F$ as an orbit in $Z$. 
Examples To fix ideas, let us describe the flag manifolds and their canonical fibrations for two simple Lie groups of rank 3 . In each case there are $7=2^{\text {rankG }}-1$ flag manifolds.

First let $G=\mathrm{SU}(4)$. There are two inner symmetric $G$-spaces: $\mathbf{C} P^{3}$ and the Grassmannian $G_{2}\left(\mathbf{C}^{4}\right)$ (of course, qua Riemannian symmetric space $G_{3}\left(\mathbf{C}^{4}\right)$ is the same as $\mathbf{C} P^{3}$ ). The flag manifolds are given by

$$
F\left(r_{1}, \ldots, r_{k} ; \mathbf{C}^{4}\right)=\frac{\mathrm{SU}(4)}{\mathrm{S}\left(\mathrm{U}\left(r_{1}\right) \times \cdots \times \mathrm{U}\left(r_{k}\right)\right)}
$$

where $r_{1}+\cdots+r_{k}=4$ and the complex structure is induced by the inclusion

$$
F\left(r_{1}, \ldots, r_{k} ; \mathbf{C}^{4}\right) \longrightarrow G_{r_{1}}\left(\mathbf{C}^{4}\right) \times G_{r_{1}+r_{2}}\left(\mathbf{C}^{4}\right) \times \cdots \times G_{r_{1}+\cdots+r_{k-1}}\left(\mathbf{C}^{4}\right) .
$$

The symmetric space associated to $F\left(r_{1}, \ldots, r_{k} ; \mathbf{C}^{4}\right)$ is $G_{\sum r_{2 i}}\left(\mathbf{C}^{4}\right)$. There is an "antipodal map" on $G_{2}\left(\mathbf{C}^{4}\right)$ given by taking the perpendicular complement of an element and so the flag manifolds that fibre canonically over $G_{2}\left(\mathbf{C}^{4}\right)$ do so twice. They are $F\left(1,1,1,1 ; \mathbf{C}^{4}\right)$, $F\left(1,2,1 ; \mathbf{C}^{4}\right)$ and $F\left(2,2 ; \mathbf{C}^{4}\right)$. Of course, $F\left(2,2 ; \mathbf{C}^{4}\right)$ is just $G_{2}\left(\mathbf{C}^{4}\right)$ itself and the fibrations are just the identity and the antipodal map. We also note that $G_{2}\left(\mathbf{C}^{4}\right)$ is quaternionic Kähler so that the discussion in section 3 applies. In particular, recall that the 2 -sphere bundle of $\mathcal{C}$ is a complex submanifold of $Z$ : in our setting this is just one of the two realisations of $F\left(1,2,1 ; \mathbf{C}^{4}\right)$ in $Z$. The remaining flag manifolds $F\left(2,1,1 ; \mathbf{C}^{4}\right), F\left(1,1,2 ; \mathbf{C}^{4}\right), F\left(1,3 ; \mathbf{C}^{4}\right)$ and $F\left(3,1 ; \mathbf{C}^{4}\right)$ fibre canonically over $\mathbf{C} P^{3}$ and may be identified with the various Grassmannian bundles $G_{r}\left(T^{1,0} \mathbf{C} P^{3}\right)$ described in section 3. In conclusion, we see that, for $\mathbf{C} P^{3}, Z$ has 4 components while, for $G_{2}\left(\mathbf{C}^{4}\right)$, it has 6 .

Now let $G=\mathrm{SO}(7)$. There are again three simply connected inner symmetric $G$-spaces: $S^{6}$; $\widetilde{G}_{3}\left(\mathbf{R}^{7}\right)$ the Grassmannian of oriented 3-planes in $\mathbf{R}^{7}$ and $\widetilde{G}_{2}\left(\mathbf{R}^{7}\right)$ the 5-quadric. All of these may be viewed as Grassmannians of oriented $k$-planes for some $k$ and so possess an antipodal map given by reversing the orientation of the $k$-planes. Thus each flag manifold has two canonical fibrations. The flag manifolds are given by

$$
F_{\text {iso }}\left(r_{1}, \ldots, r_{k} ; \mathbf{R}^{7}\right)=\frac{\mathrm{SO}(7)}{\mathrm{U}\left(r_{1}\right) \times \cdots \times \mathrm{U}\left(r_{k}\right) \times \mathrm{SO}\left(7-2 \sum r_{i}\right)}
$$

where $r_{1}+\cdots+r_{k} \leq 3$. To see the complex structure, we realise $F_{\text {iso }}\left(r_{1}, \ldots, r_{k} ; \mathbf{R}^{7}\right)$ as a family of isotropic flags in $\mathbf{C}^{7}=\left(\mathbf{R}^{7}\right)^{\mathbf{C}}$ :

$$
F_{\text {iso }}\left(r_{1}, \ldots, r_{k} ; \mathbf{R}^{7}\right)=\left\{V_{1} \subset \ldots \subset V_{k} \subset \mathbf{C}^{7}: \operatorname{dim}_{\mathbf{C}} V_{i}=r_{1}+\cdots+r_{i} ; V_{i} \text { isotropic }\right\}
$$

and then the complex structure is induced from the natural inclusion into $G_{r_{1}}\left(\mathbf{C}^{7}\right) \times \cdots \times$ $G_{r_{k}}\left(\mathbf{C}^{7}\right)$. Now set $r_{k+1}=\frac{1}{2}\left(7-\sum r_{i}\right)$ so that $2\left(r_{1}+\cdots+r_{k+1}\right)=7$ and then the symmetric space associated to $F_{\text {iso }}\left(r_{1}, \ldots, r_{k} ; \mathbf{R}^{7}\right)$ is $\widetilde{G}_{\sum 2 r_{2 i}}\left(\mathbf{R}^{7}\right)$ with the canonical fibrations induced by the inclusions

$$
\begin{aligned}
& \mathrm{U}\left(r_{1}\right) \times \cdots \times \mathrm{U}\left(r_{k}\right) \times \mathrm{SO}\left(2 r_{k+1}\right) \rightarrow \\
& \quad \mathrm{SO}\left(2 r_{1}\right) \times \cdots \times \mathrm{SO}\left(2 r_{k}\right) \times \mathrm{SO}\left(2 r_{k+1}\right) \rightarrow \mathrm{SO}\left(\sum 2 r_{2 i+1}\right) \times \mathrm{SO}\left(\sum 2 r_{2 i}\right) .
\end{aligned}
$$

We now see that $F_{\text {iso }}\left(3 ; \mathbf{R}^{7}\right)$ fibres canonically over $S^{6} ; \quad F_{\text {iso }}\left(1 ; \mathbf{R}^{7}\right), \quad F_{\text {iso }}\left(1,1 ; \mathbf{R}^{7}\right)$ and $F_{\text {iso }}\left(2,1 ; \mathbf{R}^{7}\right)$ over $\widetilde{G}_{2}\left(\mathbf{R}^{7}\right)$ and $F_{\text {iso }}\left(2 ; \mathbf{R}^{7}\right), F_{\text {iso }}\left(1,2 ; \mathbf{R}^{7}\right)$ and $F_{\text {iso }}\left(1,1,1 ; \mathbf{R}^{7}\right)$ over $\widetilde{G}_{3}\left(\mathbf{R}^{7}\right)$. We remark that $\widetilde{G}_{3}\left(\mathbf{R}^{7}\right)$ is quaternionic Kähler and that the associated sphere-bundle twistor space is one of the realisations of $F_{\text {iso }}\left(2 ; \mathbf{R}^{7}\right)$. In conclusion, for $S^{6}, Z$ has two components which are just $J_{+}\left(S^{6}\right)$ and $J_{-}\left(S^{6}\right)$ while the other two symmetric spaces have six components each in $Z$. 
There is a well-known duality between symmetric spaces of compact and non-compact type and this duality extends to the twistor theory we have been discussing. For each non-compact real form $G_{\mathrm{R}}$ of a complex semisimple group Lie group $G^{\mathbf{C}}$, there is a unique Riemannian symmetric space $G_{\mathrm{R}} / K$ of non-compact type. The corresponding involution is called the Cartan involution of $G_{\mathrm{R}}$. The above development suggests that we restrict attention to $G_{\mathrm{R}}$ with inner Cartan involution.

Consider now the orbits of such a $G_{\mathrm{R}}$ on the various flag manifolds $F=G^{\mathbf{C}} / P$. Those orbits which are open subsets of $F$ we call flag domains: in our situation, an orbit is a flag domain precisely when the stabilisers contain a compact Cartan subgroup of $G_{\mathrm{R}}$. It turns out that the presence of this compact Cartan subgroup is precisely what we need to define a canonical element of $\mathbf{g}_{R}$ and thus an involution of $\mathbf{g}_{R}$ just as in the compact case. However the involution is not necessarily a Cartan involution (i.e. the associated symmetric space need not be Riemmanian). In case that the involution is a Cartan involution we say that our flag domain is a canonical flag domain and then we exponentiate the involution to get a Riemannian symmetric space of non-compact type and a canonical fibration (unique in this case) of our canonical flag domain over it. We may now repeat the analysis of the compact case and, in particular, we find that $G_{\mathrm{R}}$ acts transitively on connected components of $Z \subset J\left(G_{\mathrm{R}} / K\right)$. Further, each component is a canonically fibred canonical flag domain so that (4.2) holds in the non-compact setting.

It is interesting to note that these flag domains have arisen in other areas: indeed, for $G^{\mathbf{C}}=$ $\mathrm{SO}(n, \mathbf{C})$ or $\operatorname{Sp}(n, \mathbf{C})$, they form a subset of the Griffiths period matrix domains [19] that classify Hodge structures. There are intriguing relationships between the theory of variation of Hodge structure and the theory of minimal surfaces in compact Riemannian symmetric spaces that arise from this twistor space duality. For example, both flag manifolds and flag domains carry an invariant holomorphic distribution which is transverse to the fibres of the canonical fibrations. This super-horizontal distribution is defined at the identity coset as the $\sqrt{-1}$-eigenspace $\mathbf{g}_{1}$ of the canonical element. It has the following interesting property:

Theorem 4.7 ([12]) Let $X$ be a flag manifold or canonical flag domain and $\pi: X \rightarrow N$ be a homogeneous fibration onto a Riemannian symmetric space. If $\phi: M \rightarrow X$ is a holomorphic map of a Kähler manifold with image tangent to the super-horizontal distribution then $\pi \circ \phi: M \rightarrow N$ is harmonic.

We call maps $\phi: M \rightarrow X$ satisfying the hypotheses of (4.7) super-horizontal holomorphic maps.

For instance, let $X=\mathrm{SU}(n+1) / \mathrm{S}(\mathrm{U}(1) \times \cdots \times \mathrm{U}(1))=F\left(1, \ldots, 1 ; \mathbf{C}^{n+1}\right)$. There are $n+1$ homogeneous fibrations $\pi_{i}: X \rightarrow \mathbf{C} P^{n}, i=1, \ldots, n$, with $\pi_{0}$ holomorphic and $\pi_{n}$ antiholomorphic. Now a super-horizontal holomorphic map $\phi: S^{2} \rightarrow X$ is essentially just the Frenet frame of the holomorphic map $\pi_{0} \circ \phi: S^{2} \rightarrow \mathbf{C} P^{n}$ while each $\pi \circ \phi$ is a harmonic map $S^{2} \rightarrow \mathbf{C} P^{n}$. It is the content of the classification theorem for harmonic 2-spheres in $\mathbf{C} P^{n}$ that all such harmonic maps arise in this way. Thus in this situation all harmonic maps are produced via (4.7).

On the non-compact side of the fence, the super-horizontal distribution is precisely that which defines the infinitesimal period relation. Thus the (local lifts of) period maps are precisely the super-horizontal holomorphic maps into period domains. Much progress has recently been made by Carleson-Toledo [13] on the relationship between period maps and harmonic maps into compact quotients of symmetric spaces of non-compact type and it seems likely that harmonic maps of Kähler manifolds into such quotients of sufficiently high rank are covered by period 
maps as in (4.7).

Finally, let us briefly describe the situation for non-inner even-dimensional Riemannian symmetric spaces. Here it can be shown that $Z$ is nonempty and, of course, by (4.1) each orbit in $Z$ is an almost complex submanifold on which $\mathcal{J}$ is integrable. However the orbits are no longer flag manifolds or flag domains and $G$ no longer acts transitively on connected components of $Z$.

\section{$5 \quad$ Flags and the loop group}

There is another realisation of the canonical fibrations of flag manifolds that serves to introduce a twistor space of a quite different type. For this, assume that $G$ is of adjoint type (i.e. has trivial centre) and let $\Omega G$ denote the infinite-dimensional manifold of based loops in $G$ : the loop group. In fact $\Omega G$ is a Kähler manifold [23] and may be viewed as a flag manifold $\mathcal{G}^{\mathrm{C}} / \mathcal{P}$ where $\mathcal{G}^{\mathbf{C}}$ is the manifold of loops in $G^{\mathbf{C}}$ and $\mathcal{P}$ is the subgroup of those that extend holomorphically to the disc $[17,23]$. We have various fibrations $\rho_{\lambda}: \Omega G \rightarrow G$ given by evaluation at $\lambda \in S^{1}$ and in some ways $\rho_{-1}$ behaves like a canonical fibration making $\Omega G$ into a universal twistor space for $G$. For instance, it is a theorem of Uhlenbeck [28] that any harmonic map of $S^{2}$ into $G$ is of the form $\rho_{-1} \circ \Phi$ for some "super-horzontal" holomorphic map $\Phi: S^{2} \rightarrow \Omega G$.

The flag manifolds of $G$ embed in $\Omega G$ as conjugacy classes of geodesics and we find a particular embedding of this kind using the canonical element. Indeed, our assumption that $G$ be centrefree means that $\exp 2 \pi \xi=e$ for any canonical element $\xi$. Thus if $F=G / H=G^{\mathbf{C}} / P$ is a flag manifold with $\xi$ the canonical element of $\mathbf{p}$, we may define a map $\Gamma: F \rightarrow \Omega G$ by setting

$$
\Gamma(e H)=\left(e^{\sqrt{-1} t} \mapsto \exp t \xi\right)
$$

and extending by equivariance. Moreover, if $N$ is the inner symmetric space associated to $F$, we have a totally geodesic immersion $\gamma: N \rightarrow G$ defined by setting $\gamma(x)$ equal to the element of $G$ that generates the involution at $x$. We now have:

Proposition 5.1 $\Gamma: F \rightarrow \Omega G$ is a totally geodesic, holomorphic, isometric immersion and the following diagram commutes

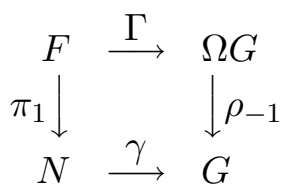

where $\pi_{1}$ is a canonical fibration.

Thus we have a third realisation of the canonical fibrations as the trace of $\rho_{-1}$ on certain conjugacy classes of geodesics. The Reader is invited to ponder on the relation between these three constructions.

\section{Conclusion}

We have seen that the construction of complex manifolds associated to a Riemannian manifold $N$ by the above methods requires stringent conditions on the curvature of $N$. However, the 
construction can be carried through for a fairly large class of geometrically interesting Riemannian manifolds. As for applications, the twistor theory of quaternionic Kähler and hyperkähler manifolds is highly developed [21, 24] while for symmetric spaces it is only just beginning (see [12], though, for applications to minimal surfaces) and there are many unanswered questions. Let us finish by mentioning a few of these.

- We have seen that there is a good theory of flag spaces fibring over Riemannian symmetric spaces. What can be said about pseudo-Riemannian symmetric spaces? Certainly, one can produce such fibrations of non-canonical flag domains.

- If we view the Riemannian symmetric spaces as "flat" examples, are there "curved" analogues of the above theory? The example of quaternionic Kähler manifolds suggests that there are far less twistor spaces in the non-symmetric case but there is still a satisfactory theory. A good test case for this would be the complex paraconformal manifolds of Bailey-Eastwood [3] which are "curved" versions of the complex Grassmannians.

- Finally, an interesting but almost certainly ill-posed question is: what is the relationship between the theory discussed in this chapter and the highly developed twistor theory of space-time discussed elsewhere in this volume?

\section{References}

[1] M. F. Atiyah, D. G. Drinfeld, N. J. Hitchin, Y. I. Manin. Construction of instantons. Phys. Lett. 65A (1978), 185-187.

[2] M. F. Atiyah, N. J. Hitchin and I. M. Singer. Self-duality in four-dimensional Riemannian geometry. Proc. Roy. Soc. Lond. A362 (1978), 425-461.

[3] T. N. Bailey and M. G. Eastwood. Complex paraconformal manifolds - their differential geometry and twistor theory. Preprint.

[4] M. Berger. Sur les groupes d'holonomie des variétés à connexion affine et des variétés riemanniennes. Bull. Soc. Math. France. 15 (1955), 279-330.

[5] A. Borel. Kählerian coset spaces of semi-simple Lie groups. Proc. Nat. Acad. Sci. USA. 40 (1954), 1147-151.

[6] A. Borel and F. Hirzebruch. Characteristic classes and homogeneous spaces, I. Amer. J. Math. 80 (1958), 459-538.

[7] R. L. Bryant. Conformal and minimal immersions of compact surfaces into the 4-sphere. J. Diff. Geom. 17 (1982), 455-473.

[8] R. L. Bryant. Lie groups and twistor spaces. Duke Math. J. 52 (1985), 223-261.

[9] D. Burns. Harmonic mappings from $\mathbf{C} P^{1}$ to $\mathbf{C} P^{n}$. In: Proc. Tulane Conf., Lecture Notes in Math. 949 (Springer, Berlin, 1982), pp. 48-56.

[10] F. E. Burstall. Twistor methods for harmonic maps. In: Differential Geometry (ed. V. L. Hansen), Lecture Notes in Math. 1263 (Springer, Berlin, 1987), pp. 55-96. 
[11] F. E. Burstall. Recent developments in twistor methods for harmonic maps. In: Harmonic mappings, twistors and $\sigma$-models (ed. P. Gauduchon, World Scientific, Singapore, 1988), pp. $158-176$.

[12] F. E. Burstall and J. H. Rawnsley. Twistor theory for Riemannian symmetric spaces with applications to harmonic maps of Riemann surfaces. (preprint) 1989.

[13] J. A. Carleson and D. Toledo. Harmonic mappings of Kähler manifolds to locally symmetric spaces. (Utah preprint) 1988.

[14] A. M. Din and W. J. Zakrzewski. General classical solutions in the $\mathbf{C} P^{n-1}$ model. Nucl. Phys. B. 174 (1980), 397-406.

[15] M. Dubois-Violette. Structures complexes au-dessus des variétés. In: Mathématiques et Physique Progress in Math. 37 (Birkhauser, Boston, 1983).

[16] J. Eells and J. C. Wood. Harmonic maps from surfaces to complex projective spaces. Adv. in Math. 49 (1983), 217-263.

[17] D. S. Freed The geometry of loop groups. J. Diff. Geom. 28 (1988), 223-276.

[18] V. Glaser and R. Stora. Regular solutions of the $\mathbf{C} P^{n}$ models and further generalisations. CERN preprint, 1980.

[19] P. A. Griffiths. Periods of integrals on algebraic manifolds, III. Publ. Math. I.H.E.S 38 (1970), 125-180.

[20] N. J. Hitchin. Monopoles and geodesics. Comm. Math. Phys. 83 (1982), 579-602.

[21] N. J. Hitchin, A. Karlhede, U. Lindström and M. Roček. Hyperkähler metrics and supersymmetry. Comm. Math. Phys. 108 (1987), 535-589.

[22] N. R. O'Brian and J. H. Rawnsley. Twistor spaces. Ann. Glob. Anal. and Geom. 3 (1985), $29-58$.

[23] A. N. Pressley and G. Segal. Loop Groups. Oxford Math. Monographs, 1986.

[24] S. M. Salamon. Quaternionic Kähler manifolds. Invent. Math. 67 (1982), 143-171.

[25] S. M. Salamon Riemannian Geometry and holonomy groups. Pitman Research Notes in Math. 201, 1989.

[26] I. M. Singer and J. A. Thorpe. The curvature of 4-dimensional Einstein spaces. In: Global Analysis, Papers in honor of K. Kodaira (ed. D. C. Spenser and S. Iyanaga, Princeton University Press, New Jersey, 1969), pp. 355-365.

[27] A. J. Small. A twistorial interpretation of the Weierstrass representation formulae. Ph.D. thesis. University of Warwick 1989.

[28] K. Uhlenbeck. Harmonic maps into Lie groups (classical solutions of the chiral model). J. Diff. Geom. 30 (1989), 1-50.

[29] J. C. Wood. Twistor constructions for harmonic maps. In: Differential Geometry and Differential Equations (eds. C. H. Gu, M. Berger and R. L. Bryant.) Lecture Notes in Math. 1255 (Springer, Berlin, 1988), pp. 130-159.

[30] R. S. Ward. On self-dual gauge fields. Phys. Lett. 61A (1977), 81-82. 
[31] J. A. Wolf. The action of a real semi-simple Lie group on a complex flag manifold. I: Orbit structure and holomorphic arc components. Bull. Amer. Math. Soc. 75 (1969), 1121-1237. 